\title{
Experimental and simulation study of the Schottky barrier lowering by substrate doping variation for PtSi Source/Drain SBFETs
}

\author{
Gregory P. Lousberg ${ }^{1}$, H.Y. Yu*, B. Froment ${ }^{2}$, M.F. Li ${ }^{3}$, E. Augendre, A. De Keersgieter, C. Demeurisse, S. Brus, \\ B. Degroote, T. Hoffmann, A. Lauwers, M. DePotter, S. Kubicek, K. Anil, P. Absil, M. Jurczak, S. Biesemans \\ IMEC, Kapeldreef 75, B-3001, Leuven, Belgium, ${ }^{1}$ Univ. of Liege, Belgium, ${ }^{2}$ STM assignee to IMEC, ${ }^{3}$ Dept. ECE/ NUS, Singapore \\ *hongyu@imec.be; tel: 0032-16288613; fax: 0032-16281706
}

\begin{abstract}
In this paper, we study experimentally and numerically the Schottky barrier height (SBH) lowering of Pt silicide/n-Si diodes and its implications to Schottky-barrier (SB) source/drain $p$-FETs. We demonstrate that hole SBH can be lowered through an image-force mechanism by increasing the $n$-Si substrate doping, which leads to a substantial gain of the drive current in the long-channel bulk $p$-SBFETs. Numerical simulations show that the channel doping concentration is also critical for short-channel $n$ - \& p- SOI SBFETs performance.
\end{abstract}

\section{INTRODUCTION}

Silicide source/drain (S/D) Schottky barrier field-effect transistors (SBFETs) are promising candidates for sub- $32 \mathrm{~nm}$ CMOS technology [1-4], as the silicide S/D can provide abrupt junctions together with lower serial resistance as compared to conventional doped S/D junctions. One of the key challenges in SBFETs is to obtain a low Schottky barrier height $(\mathrm{SBH})$ at the silicide-channel junction [2]-[3]. $\mathrm{PtSi} / \mathrm{YbSi}_{1+\mathrm{x}}$ were considered to be among the best silicide materials for $p$-/ $n$ - SBFETs application, due to their relative low hole/ electron SBH [4].

Recently, different approaches have been proposed to reduce the electron $\mathrm{SBH}$, e.g. by inserting a thin insulator layer [3] or by creating a highly doped region located at the silicide/Si interface with a dopant segregation technique [5][6]. To our knowledge, no such reports were available for hole SBH modulation. In this paper, we experimentally demonstrate that hole $\mathrm{SBH}$ of $\mathrm{PtSi} / n-\mathrm{Si}$ diodes could be lowered through an image-force mechanism [7] by increasing the $n$-Si substrate doping, which leads to a substantial gain of the drive current in the long-channel bulk $p$-SBFETs. Numerical simulations show that the substrate

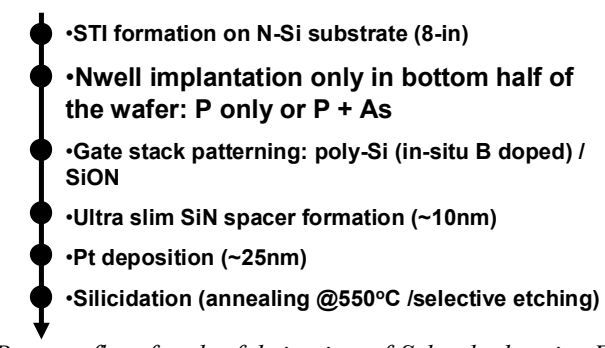

Figure 1. Process flow for the fabrication of Schottky barrier FET. Gate length and gate with are $1 \mu \mathrm{m}$. (channel) doping is also critical for short-channel bulk \& SOI SBFETs performance improvement. SBH modulation method proposed in this work only relies on the conventional CMOS process techniques such as well ion implantation $(\mathrm{I} / \mathrm{I})$, and thus is very attractive for future SBFETs development.

\section{DEVICE FABRICATION}

The SBFETs device $(\mathrm{W} / \mathrm{L}=1 \mu \mathrm{m} / 1 \mu \mathrm{m})$ fabrication process is depicted in Fig. 1. Note that only bottom part of the wafers received the well $\mathrm{I} / \mathrm{I}$ : either $\mathrm{P}\left(3 \mathrm{e} 12 \mathrm{~cm}^{-2}, 120 \mathrm{KeV}\right)$ or $\mathrm{P}$ $\left(3 \mathrm{e} 12 \mathrm{~cm}^{-2}, 120 \mathrm{KeV}\right)+$ As $\left(1 \mathrm{e} 12 \mathrm{~cm}^{-2}, 90 \mathrm{KeV}\right)$. Here we define bottom part as south well, and top part as north well in the following text. The simulated dopant profile of the south well with the 2 different splits is reproduced in Fig. 2 (a). CV measurements of MOS capacitors (data not shown) indicates a substrate doping level of $1 \mathrm{e} 16 \mathrm{~cm}^{-3}$ in the north well and of $8 \mathrm{e} 16$ or $2 \mathrm{e} 17 \mathrm{~cm}^{-3}$ in the south well respectively with $\mathrm{P}$ or $\mathrm{P}+\mathrm{As} \mathrm{I} / \mathrm{I}$. The EOT of SiON extracted from $\mathrm{CV}$ for the SBFETs is $\sim 2 \mathrm{~nm}$. XSEM of a SBFET with PtSi S/D ( $\sim 50 \mathrm{~nm}$ in depth, consistent with $1 \AA \mathrm{Pt}+1.32 \AA \mathrm{Si}->2.0 \AA$ PtSi) is presented in Fig. 2 (b). A slim SiN spacer $(\sim 11 \mathrm{~nm})$ is used in our work to enhance the device drive current [8]. XSEM shows that the S/D silicide reaches the gate edge due to a lateral diffusion of the PtSi under the SiN spacer.
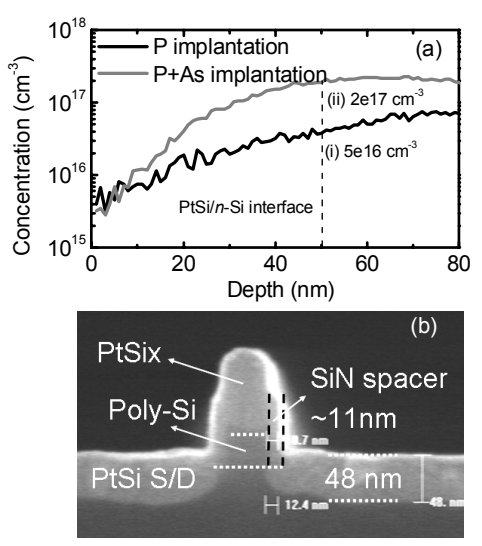

Figure 2. (a) Dopant concentration in the south well in the case of $3 e 12$ $\mathrm{cm}^{-2}-120 \mathrm{KeV} \mathrm{P}$ implantation and $3 e 12 \mathrm{~cm}^{-2}-120 \mathrm{KeVP}+1 e 12 \mathrm{~cm}^{-2}-$ $90 \mathrm{KeV}$ As implantation. The PtSi/n-Si interface is located at $48 \mathrm{~nm}$ in depth. (b) XSEM of the SBFET. 


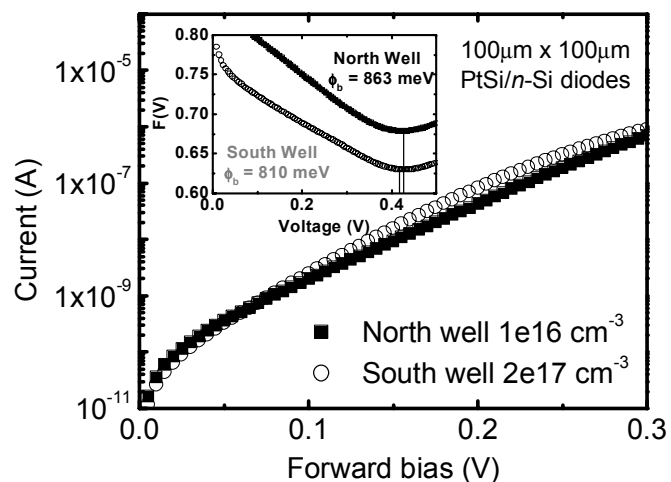

Figure 3. IV characteristics of PtSi/n-Si diodes. The substrate doping is $1 e 16 \mathrm{~cm}^{-3}$ (square) and $2 e 17 \mathrm{~cm}^{-3}$ (circle). Inset shows the corresponding Norde function $F(V)$. Extracted electron barriers $\left(\phi_{b}\right)$ are $863 \mathrm{meV}$ (north well) and $810 \mathrm{meV}$ (south well).

\section{EXTRACTION OF SBH}

We use the diode forward current-voltage (I-V) for the SBH extraction [7]. Fig. 3 shows typical I-V curves measured from two diodes $(100 \mu \mathrm{m} \times 100 \mu \mathrm{m})$ in wells with different dopant concentration. Considering the possible high series resistance, we adapted a method initially given by Norde[9]. The electron SBH $\left(\phi_{\mathrm{e}}\right)$ was thus extracted from the minimum value of the Norde function $\mathrm{F}(\mathrm{V})$ (inset of Fig.3)

$$
F(V)=\frac{V}{2}-\frac{k T}{q} \ln \left(\frac{I}{A A^{* *} T^{2}}\right) \Rightarrow \phi_{e}=F\left(V_{\min }\right)+\frac{V_{\min }}{2}-\frac{k T}{q}
$$

A set of extracted electron $\mathrm{SBH}$ of $\mathrm{PtSi} / \mathrm{n}-\mathrm{Si}$ diodes is plotted in Fig. 4. SB diodes were measured in a statistical manner, i.e. they lie on a north-to-south straight line in the wafer. Comparison of the SBH extracted w/ or w/o considering Norde model [Fig. 4 (a)-(b)] justifies the Norde model used in this work for SBH extraction. In Fig. 4 (b), it is seen that the electron $\mathrm{SBH}$ distribution of the north well diodes is tight and has an average value close to $870 \mathrm{meV}$. In the south well, the values of the SBH are a function of the doping concentration: the average value is around $840 \mathrm{meV}$ for the $8 \mathrm{e} 16 \mathrm{~cm}^{-3}$ doped substrate and $820 \mathrm{meV}$ when the doping concentration is $2 \mathrm{e} 17 \mathrm{~cm}^{-3}$. Note that the extracted SBHs show more fluctuation in the south well. The PtSi thickness variations in the non-uniformly doped substrate [see Fig. 2 (a)] are suspected to be the reason for these SBH fluctuations. It is expected that they could be minimized by improving PtSi thickness variations.

The electron SBH is reduced by $\sim 30 \mathrm{meV}$ and $\sim 50 \mathrm{meV}$ respectively when the doping level rises from $1 \mathrm{e} 16 \mathrm{~cm}^{-3}$ to $8 \mathrm{e} 16 \mathrm{~cm}^{-3}$ and $2 \mathrm{e} 17 \mathrm{~cm}^{-3}$. This lowering may be caused by the electrostatic image-force attracting the carriers to the metal and therefore decreasing the electric field repealing the carrier from the interface [7]. As sketched in Fig. 5, this would result in a reduction of the effective electron and hole $\mathrm{SBH}\left(\phi_{\mathrm{e} 0}\right.$ and $\left.\phi_{\mathrm{h} 0}\right)$. According to the image-force lowering

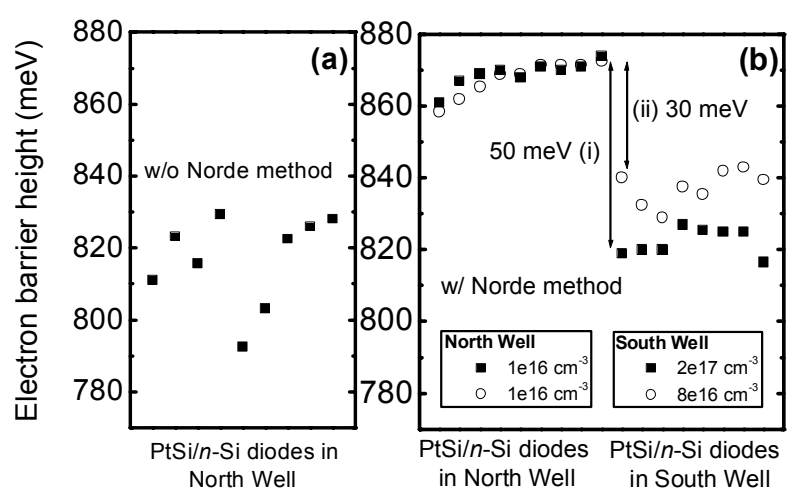

Figure 4. Electron barrier heights extracted without (a) and with (b) Norde method of PtSi/n-Si diodes located on a straight line in the wafer. The n-type bulk Si substrate has a dopant concentration of 1 e16 cm-3 (north well), $8 e 16 \mathrm{~cm}-3$ (circle in south well) and $2 e 17 \mathrm{~cm}-3$ (square in south well).

model $\left(\Delta \phi=2 \sqrt{q E_{\max } / 16 \pi \varepsilon_{s}}\right)$ [7], we have calculated from MEDICI simulation [10] the SBH lowering corresponding to the maximum electric field value at the interface $\left(E_{\max }\right)$, and the data are summarized in Table 1. It is seen that the simulations are in excellent agreement with our measurement data. We should also note that the electric field increases with the forward voltage, and thus the effective SBH lowering calculated for relatively low voltage in Table 1 would be enhanced.

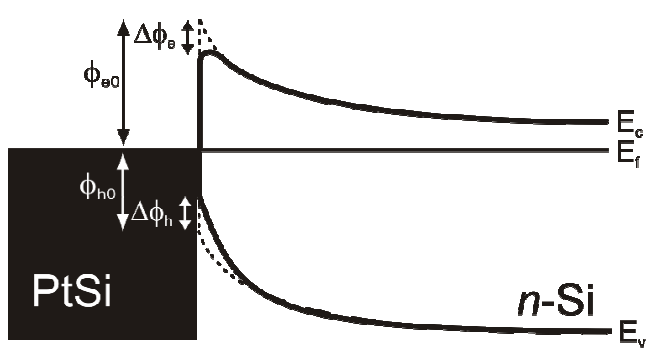

Figure 5. Image-force $S B H$ lowering $\Delta \phi_{e}$ and $\Delta \phi_{h}$ for the electron barrier height $\phi_{e 0}$ and hole barrier height $\phi_{h 0}[7]$

\begin{tabular}{c|c|c|c|c}
$\begin{array}{c}\text { Doping } \\
\left(\mathbf{c m}^{-3}\right)\end{array}$ & $\begin{array}{c}\boldsymbol{E}_{\max } \text { at } \\
\text { interface } \\
(\mathbf{V} / \mathbf{c m})\end{array}$ & $\begin{array}{c}\boldsymbol{e} \text {-SBH } \\
\text { lowering by } \\
\text { image-force }\end{array}$ & $\begin{array}{c}\text { Calculated } \\
\boldsymbol{e}-\text { SBH } \\
\text { difference }\end{array}$ & $\begin{array}{c}\text { Measured } \\
\boldsymbol{e} \text {-SBH } \\
\text { difference }\end{array}$ \\
\hline $1 \mathrm{e} 16$ & $2 \mathrm{e} 4$ & $15.7 \mathrm{meV}$ & - & - \\
\hline $8 \mathrm{e} 16$ & $1 \mathrm{e} 5$ & $39.3 \mathrm{meV}$ & $\sim 25 \mathrm{meV}$ & $30 \mathrm{meV}$ \\
\hline $2 \mathrm{e} 17$ & $3 \mathrm{e} 5$ & $60.9 \mathrm{meV}$ & $\sim 45 \mathrm{meV}$ & $50 \mathrm{meV}$
\end{tabular}

Table 1. Simulated maximum electric field at the PtSi/n-Si junction, calculated electron SBH lowering by [7] and the calculated \& measured [from Fig. 4 (b)] e-SBH difference from the 1 el $\mathrm{cm}^{-3}$ doping level, for dopant concentrations of 1 e $16 \mathrm{~cm}^{-3}, 8 e 16 \mathrm{~cm}^{-3}$ and $2 \mathrm{e} 17 \mathrm{~cm}^{-3}$. 


\section{IMPACT OF THE BARRIER HEIGHT LOWERING ON SBFETS}

The hole SBH lowering due to the image-force mechanism (see Fig. 5) would be expected to directly impact the performance of $p$-SBFET. The $I_{s^{-}} V_{g}$ and $I_{d^{-}} V_{g}$ of the $p$ SBFETs in the north well (1 $16 \mathrm{~cm}^{-3}$, circle symbols) and the south well $\left(2 \mathrm{e} 17 \mathrm{~cm}^{-3}\right.$, square symbols) are represented in Fig. 6 (a)-(b) respectively. Due to the drain junction leakage induced by the electron tunnelling, the $I_{d, o f f}$ is much larger than $I_{s, o f f}$. This leakage could be drastically reduced by using a SOI substrate [11]. For convenience of comparison, we will focus on the $I_{s}-V_{g}$ analysis [Fig. 6 (a)]. The subthreshold slope $S S$ of $I_{s}-V_{g}$ of the SBFETs in the south well is $\sim 70$ $\mathrm{mV} /$ dec, with a $\mathrm{I}_{\text {on }} / \mathrm{I}_{\text {off }}$ ratio $\sim 10^{7}$, demonstrating excellent electrical properties of PtSi S/D SBFETs fabricated in this work. Note that the DIBL value is reduced substantially with a high substrate doping (from $40 \mathrm{mV} / \mathrm{V}$ for $1 \mathrm{e} 16 \mathrm{~cm}^{-3}$ to $5 \mathrm{mV} / \mathrm{V}$ for $2 \mathrm{e} 17 \mathrm{~cm}^{-3}$ ). The reason behind is believed to be the much reduced lateral depletion region in the channel with high substrate doping.

In Fig. 7, after considering the SBH lowering effect, MEDICI simulation well matches the measured $I_{s}-V_{g}$ of the SBFETs with a substrate doping of $1 \mathrm{e} 16 \mathrm{~cm}^{-3}(\mathrm{~b})$ and $2 \mathrm{e} 17$ $\mathrm{cm}^{-3}(\mathrm{a})$. The relative difference of $\mathrm{SBH}$ used for $I_{s}-V_{g}$ simulation is again in good agreement with the $50 \mathrm{meV}$ observed in the SBH extracted from the diode characteristic [Fig. 4 (b) \& Table 1]. Thus, we believe the image-force barrier lowering effect [7] is responsible for the electron / hole $\mathrm{SBH}$ reduction observed in diode $I-V /$ transistor $I_{s}-V_{g}$ measurement respectively. However, we should mention that the model [7] predicts a slightly larger electron SBH lowering than the corresponding hole SBH lowering.

It is interesting to note the 2 different $S S$ values measured in the $I_{s}-V_{g}$ of the transistor in the north well, while there is only $1 S S$ for the one in south well. From simulation results shown in Fig. 8, it is observed that there is a change of the SS due to the high $\mathrm{SBH}$, as $\mathrm{SBH}$ lowering modifies the curvature of the $I_{s}-V_{g}$ curve at the transition from the subthreshold thermoionic emission to the field emission regime. Thus, it is believed that the increased effective $\mathrm{SBH}$ accounts for the 2 different $S S$ observed in Fig. 7(b), confirming the previous report [11].
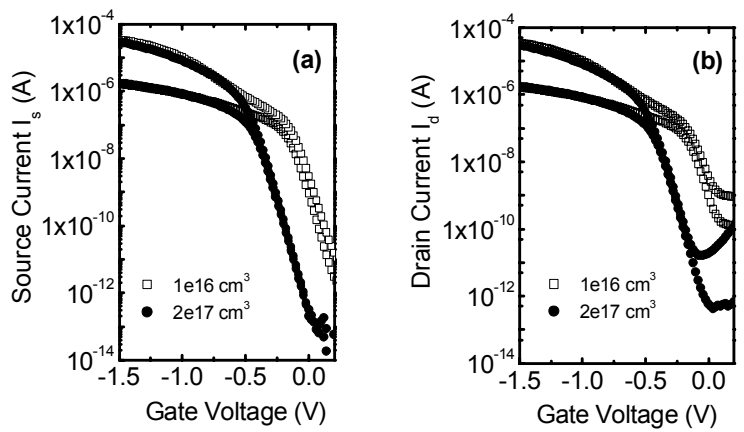


SBFET at $V_{d}=-0.05 \mathrm{~V}$ and $-1 \mathrm{~V}$, on a substrate doping of 1 el6 (square) and $2 e 17 \mathrm{~cm}^{-3}$ (circle). The source current is represented in the left panel (a) and the drain current in the right panel (b).



Figure 7. Experimental (circles) and simulated (solid lines) $I_{s^{-}} V_{g}$ of $p$ SBFET with channel doping of $2 e 17 \mathrm{~cm}^{-3}$ (left panel) and $1216 \mathrm{~cm}^{-3}$ (right panel). The hole barrier heights are respectively $190 \mathrm{meV}$ for doping concentration of $2 \mathrm{e} 17 \mathrm{~cm}^{-3}$ and $240 \mathrm{meV}$ for $1 \mathrm{e} 16 \mathrm{~cm}^{-3} . V_{d}=-1.1 \mathrm{~V}$

In Fig. 9, the measured output curve $\left(I_{s}-V_{d}\right)$ for SBFETs (a) $\left.V_{g}-V_{t}=-1 \mathrm{~V}\right)$ with 3 different substrate doping demonstrates the substantial gain of the drive current due to the high doping stemming from the SBH lowering effect. Note that a reduction of the hole mobility due to high substrate impurities tends to lower the drive current, but the overall effect of increasing substrate doping is to enhance the drive current through the image-force barrier lowering effect.

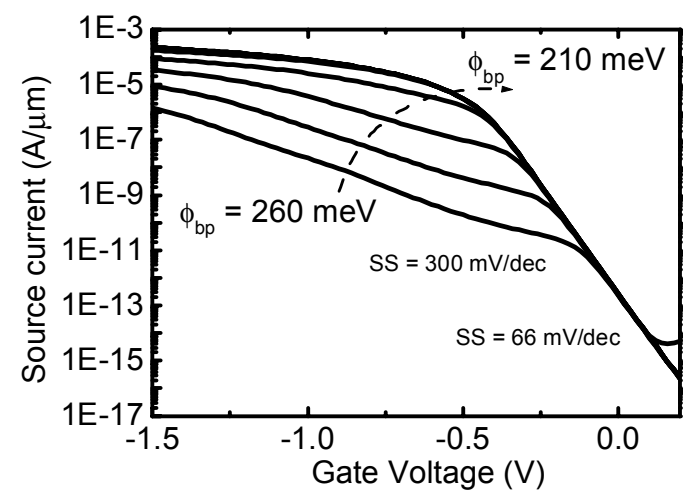

Figure 8. Simulated transfer characteristics of a 1 4 m-channel length SSDT for hole barrier heights varying from $210 \mathrm{meV}$ to $260 \mathrm{meV}$. Doping concentration is 1 e $16 \mathrm{~cm}^{-3}$. Drain voltage is $-1.1 \mathrm{~V}$.

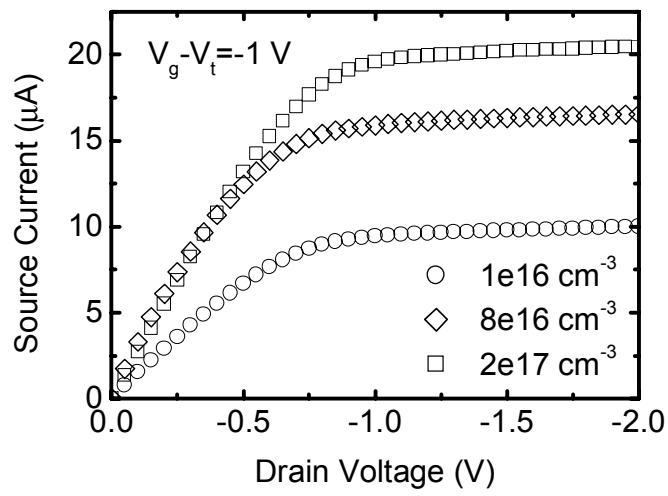

Figure 9. Measured $I_{s^{-}} V_{d}$ of a $1 \mu m$-channel length SBFET on a substrate doping of $1216 \mathrm{~cm}^{-3}$ (circle), $8016 \mathrm{~cm}^{-3}$ (rhombus) and $2 e 17 \mathrm{~cm}^{-3}$ (square). $V_{g}-V_{t}=-1 V . V_{t}$ is defined as the voltage corresponding to a current of $10^{-8} \mathrm{~A}$ 


\section{EXTENSION TO SHORT-CHANNEL SBFETS}

The substrate doping concentration is also critical for the short channel SBFET performance. The simulated $I_{s}-V_{g}$ (after offsetting the $V_{t}$ difference, Fig. 10) of two SBFETs (with $L_{g}=100 \mathrm{~nm}$, and substrate doping of $1 \mathrm{e} 16$ and $1 \mathrm{e} 18$ $\mathrm{cm}^{-3}$ respectively) indicates higher drive current in the highly-doped SBFET, after considering the barrier lowering effect in MEDICI simulation. The similar simulation of two $100 \mathrm{~nm}$ channel length SBFETs on $\mathrm{p}$ - \& $\mathrm{n}$ - SOI substrate brings identical conclusions [Fig. 11 (a)-(b)]. The observed relative larger $I_{s}$ difference in bulk SBFET compared to the SOI one might be due to a device geography difference. Nevertheless, higher substrate doping is beneficial for device performance, on both bulk and SOI substrates.

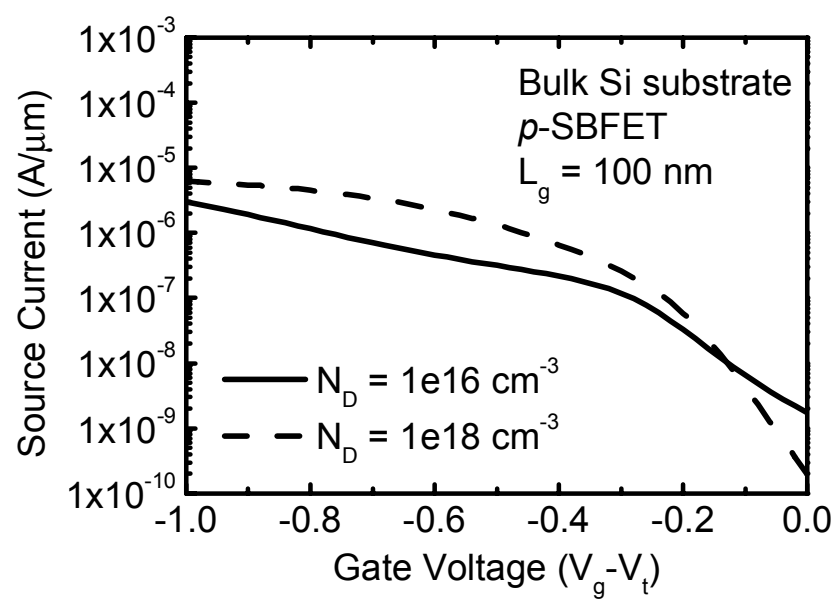

Figure 10. Simulated transfer characteristics of a $100 \mathrm{~nm}$-channel length PtSi S/D SBFET at $V_{d s}=-0.1 \mathrm{~V}$ on a bulk substrate with a doping of 1 el6 $\mathrm{cm}^{-3}$ (solid line) and $1 \mathrm{e} 18 \mathrm{~cm}^{-3}$ (dashed line).

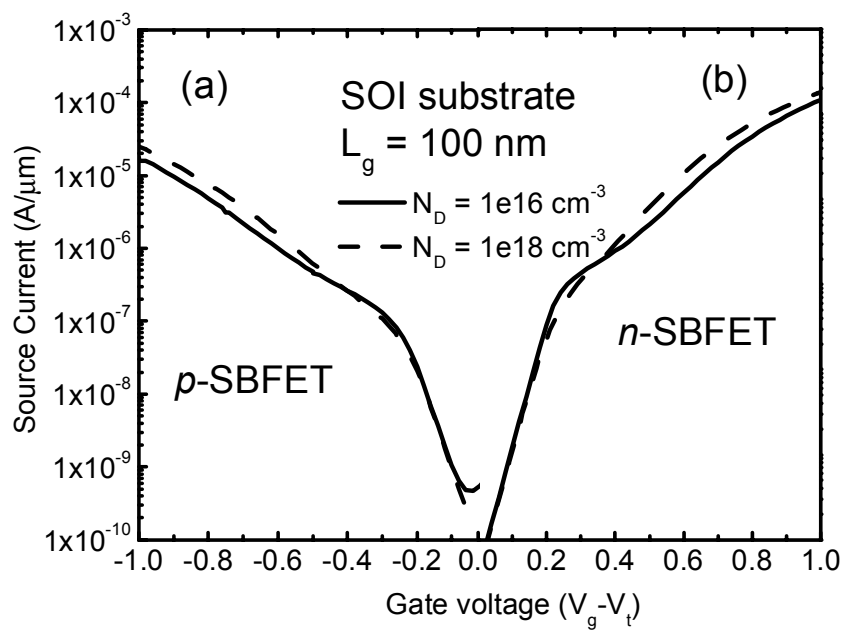

Figure 11. Simulated transfer characteristics of a $100 \mathrm{~nm}$-channel length PtSi (a) and $\mathrm{YbSi}_{1+x}$ (b) SBFET at $V_{d}=-1.1 \mathrm{~V}$ on a SOI substrate with a Si body doping of $1 e 16 \mathrm{~cm}^{-3}$ (solid line) and 1 e18 $\mathrm{cm}^{-3}$ (dashed line). Si body thickness is $10 \mathrm{~nm}$ and silicide thickness is $4 \mathrm{~nm}$.

\section{CONCLUSION}

In conclusion, we demonstrate that hole SBH can be lowered through an image-force mechanism by increasing the $n$-Si substrate doping, which leads to a substantial gain of the drive current in the long-channel bulk $\mathrm{PtSi} \mathrm{S} / \mathrm{D} p$ SBFETs. Numerical simulations show that the channel doping concentration is also beneficial for short-channel bulk $\&$ SOI $n$ - / $p$ - SBFETs. It would be critical to adjust the well I/I for SOI SBFETs as well in order to improve its performance.

\section{REFERENCES}

[1] J. Kedzierski, P. Xuan, E. Anderson, J. Bokor, T-J. King, and C. Hu, "Complementary silicide source/drain thin-body MOSFETs for the 20nm gate length regime", IEDM Technical digest 2000, pp. 57-60.

[2] C. Wang, J. P. Snyder, and J. R. Tucker, "Sub-40nm PtSi Schottky source/drain metal-oxide-semiconductor field-effect transistors", Appl. Phys. Lett., 74(8), pp. 1174-1176, 1999.

[3] D. Connelly, C. Faulkner, D. E. Grupp , and J. S. Harris, "A new route to zero-barrier metal source/drain MOSFETs", IEEE Trans. on Nanotech., 3 (1), pp. 98-104, 2004.

[4] S. Zhu, H. Y. Yu, S. J. Whang, J. H. Chen, C. Shen, et al., "Schottkybarrier S/D MOSFETs with high-K gate dielectrics and metal-gate electrode", IEEE Electr. Device Lett., 25(5), pp. 268-270, 2004.

[5] Q. T. Zhao, U. Breuer, E. Rije, St. Lenk, and S. Mantl, "Tuning of $\mathrm{NiSi} / \mathrm{Si}$ Schottky barrier heights by sulfur segregation during $\mathrm{Ni}$ silicidation", Appl. Phys. Lett., 86, 062108, 2005.

[6] J. Knoch, M. Zhang, Q. T. Zhao, St. Lenk, S. Mantl, and J. Appenzeller, "Effective Schottky barrier lowering in silicon-oninsulator Schottky-barrier metal-oxide-semiconductor field-effect transistors using dopant segregation", Appl. Phys. Lett., 87, 263505, 2005

[7] E. H. Rhoderick, Metal-Semiconductor Contacts, Clarendon, Oxford, 1978, pp. 35-38.

[8] M. Nishisaka, S. Matsumoto, and T. Asano, "Schottky source/drain SOI MOSFET with shallow doped extension", Jpn. J. Appl. Phys. 42, pp. 2009-2013, 2003.

[9] H. Norde, "A modified forward I-V plot for Schottky diodes with high series resistance”, J. Appl. Phys., 50 (7), pp. 5052-5053, 1979.

[10] MEDICI incorporates a model for calculating transport current through a Schottky contact (thermoionic and field emission) in addition to the usual MOSFET models (such as the concentrationdependant mobility). It also includes a barrier height lowering model. http://www.synopsys.com/products/tcad/taurus_medici_ds.html

[11] J. Knoch, and J. Appenzeller, "Impact of the channel thickness on the performance of Schottky barrier metal-oxide-semiconductor fieldeffect transistors", Appl. Phys. Lett., 81(16), pp. 3082-3084, 2002. 